\title{
Amiodarone-induced bronchiolitis obliterans organizing pneumonia in patient following percutaneous transluminal coronary angioplasty
}

\author{
Massimo Bolognesi ${ }^{1^{*}}$, Diletta Bolognesi ${ }^{2}$ \\ ${ }^{1}$ General Practice Medicine-Primary Care, Cesena, Italy; *Corresponding Author: massbolo1@tin.it \\ ${ }^{2}$ Territorial Medicine-Primary Care, Cesena, Italy
}

Received 23 September 2013; revised 20 October 2013; accepted 18 November 2013

Copyright (C) 2013 Massimo Bolognesi, Diletta Bolognesi. This is an open access article distributed under the Creative Commons Attribution License, which permits unrestricted use, distribution, and reproduction in any medium, provided the original work is properly cited. In accordance of the Creative Commons Attribution License all Copyrights (c) 2013 are reserved for SCIRP and the owner of the intellectual property Massimo Bolognesi, Diletta Bolognesi. All Copyright (c) 2013 are guarded by law and by SCIRP as a guardian.

\section{ABSTRACT}

Background: Many patients are affected by idiopathicbronchiolitis obliterans organizing pneumonia (BOOP). There are several known causes of BOOP, and several systemic disorders have BOOP as an associated primary pulmonary lesion. Numerous agents including cytotoxic and noncytotoxic drugs have the potential to cause pulmonary toxicity. Descriptions of amiodaronerelated BOOP continue to be reported throughout the world. Case Report: We reported a patient with original clinical presentation who developed recurrent sustained ventricular tachycardia (SVT) despite the presence of implantable cardioverter-defibrillator (ICD), hypoxaemia and interstitial pneumonitis in both lung bases. After percutaneous transluminal coronary angioplasty, he developed bronchiolitis obliterans organizing pneumonia (BOOP). Conclusions: To our knowledge, such complications after percutaneous coronary procedure in patients with amiodarone therapy for arrhythmia prophylaxis, are not very frequent in literature.

Keywords: Percutaneous Transluminal Coronary Angioplasty; Amiodarone; BOOP

\section{INTRODUCTION}

Bronchiolitis obliterans organizing pneumonia (BOOP) is a distinct entity with various clinical, radiographic and histologic features [1]. The term Bronchiolitis obliterans organizing pneumonia was first described in the early 1980s as a clinical pathologic syndrome characterized symptomatically by subacute and chronic respiratory illness, histopathologically by granulation tissue in the bronchiolar lumen, alveolar ducts with some alveoli associated with a variable degree of interstitial and air space infiltration by mononuclear cells with foamy macrophages [2]. In most cases, the aetiology remains unknown, although it has been associated with specific diseases and causes including bacterial or viral infections, diseases of the connective tissue, radiation therapy, myelodysplastic syndrome, cocaine abuse, human immunodeficiency virus (HIV) infection, gastrointestinal disorders, coronary artery bypassing grafting, and more various pharmaceutical drugs [3]. Amiodarone is one of the principal drugs involved in pulmonary toxicity, especially in patients undergoing cardiac surgery [4]. The manifestations of pulmonary toxicity from amiodarone, described in the literature include bronchiolitis obliterans with or without signs of organizing pneumonia, with or without chronic interstitial fibrosis, pulmonary solitary or multiple masses or respiratory distress syndrome [511]. A tissue biopsy specimen is needed for a precise diagnosis, but clinicoradiologic characteristics determined through biopsy-based studies may provide sufficient diagnostic information. In fact, the chest radiograph showed the typical bilateral patchy (alveolar) infiltrate and even more, the chest computed tomographic scan showed the same findings, with bilateral areas of consolidation and ground glass opacities, usually with a peripheral location [6]. High-resolution chest computed tomographic scans showed two types of linear opacities that usually occurred in the lower lobes, frequently associated with multifocal areas of consolidation, and usually completely resolved with treatment [7]. We report here a 
case of a patient with classic clinical and radiological description of amiodarone-induced BOOP seen immediately after percutaneous coronary intervention (PCI).

\section{CASE REPORT}

This report concerns an 81-year-old man suffering from ischemic dilated cardiomyopathy and permanent atrial fibrillation. The patient had had previous myocardial infarction and sustained ventricular tachycardia (SVT) with impairment of theleft ventricular systolic function that required multiple drugs, including amiodarone, and implantable cardioverter-defibrillator (ICD). Significant multivessel coronary artery disease with severe left ventricular dysfunction, estimate by ejection fraction to $30 \%$, requireda percutaneous coronary intervention (PCI) by dualangioplasty with drug-eluting stent. This procedure was carried out under poor clinical conditions. This patient was in long term treatment with L-tiroxina for jatrogenic hypothyroidism. After discharge, at home, he developed symptoms of an upper respiratory infection, worsened shortness of breath and cough and was again admitted to hospital. Postero-anterior chest radiograph recorded the first time in supine position (see Figure 1, Panel B) and 2 days later in sitting position (see Figure 1, Panel A), showed a worsening of infiltration of the bilateral inferior lobe of the lungs with mild pleural effusion. Pneumonia was initially diagnosed and an antibiotic therapy (Levofloxacina and Ceftriaxone) was started, while a cardiac ablation was performed for recurrent SVT (see Figure 2). After four days the patient remained symptomatic despite antibiotics and symptomatic management. A subsequent chest high-resolution computed tomography (HRCT) scan (see Figure 3) showed extensive bilateral opacities which were more pronounced in the lower lobes, particularly in the right lobe. In addition to the chest HRCT, which confirmed the bilateral presence of basal pulmonary infiltrates, the pulmonary func- tion tests showed reduced. Then, amiodaronew as discontinued and the patient began an anti-inflammatory treatment with steroids at high dose for presumed BOOP. Other medications such ascarvedilol, furosemide, ACE inhibitors, digoxin, ceftriaxone and nebulised bronchodilators were continued. There was a dramatic improvement in the clinical as well as radiological status within 72 hours, and a chest $x$ ray showed fairly good resolution of infiltrates (see Figure 4). Thus, the presumptive diagnosis of acute amiodarone toxicity was confirmed.

\section{CONCLUSIONS}

Pulmonary drug toxicity is a common and possibly underdiagnosed cause of acute and chronic lung disease [8]. There are numerous drugs with potential toxic effects on the lungs: one of these is amiodarone. As Nacca et al. describes in our case study [9], diagnosis of amiodarone pulmonary toxicity is often one of exclusion as there are no specific laboratory analyses to confirm this. Therefore, the diagnosis is based on a combination of clinical suspicion, history, radiographic and clinical evidence, with the exclusion of alternative etiologies. Chest $\mathrm{X}$ ray and subsequent chest HRCT show bilateral diffuse or patchy infiltrates, more commonly in the right lobe. Furthermore, pleural thickening and/or effusion has been described. Pulmonary function tests typically reveal either a restrictive or mixed obstructive/restrictive pattern with a decreased diffusion lung capacity of $15 \%$ $20 \%$ [10]. In fact, the earliest abnormality in amiodarone pulmonary toxicity is a decrease in the diffusion capacity for carbon monoxide. The aim of this report is to emphasize the possibility of this dangerous disease in patients undergoing cardiovascular surgery or other procedures such as percutaneous coronary angioplasty, especially when they are in long term therapy with amiodarone, and when signs of iatrogenic effects such as hypothyroidismare already present. The clinician must keep

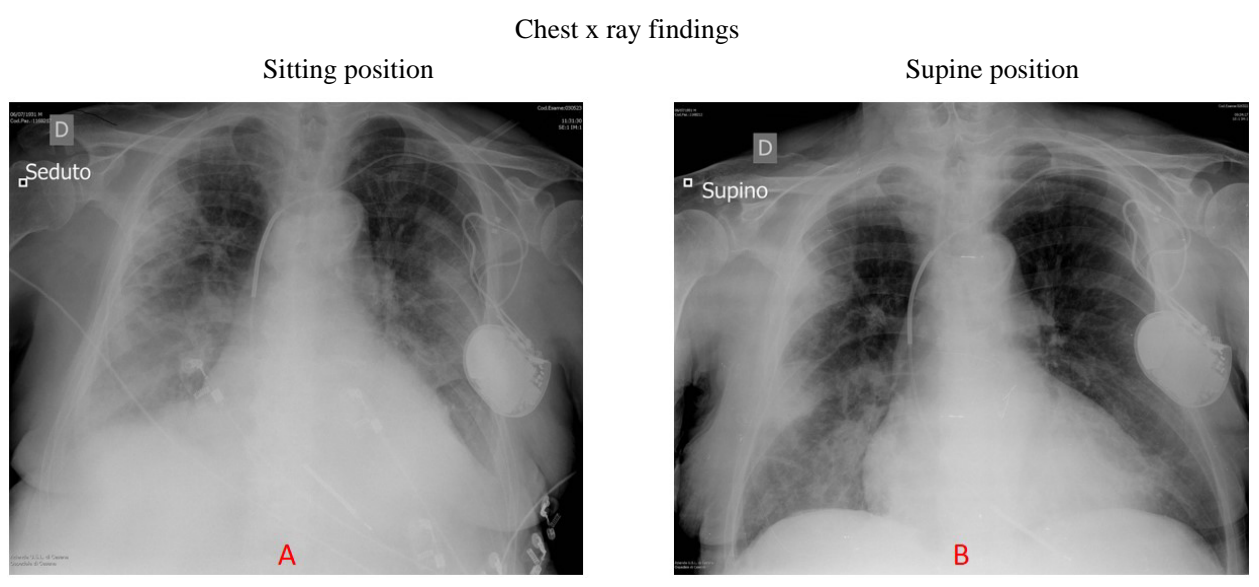

Figure 1. Chest $x$ ray, in supine (Panel B) and sitting positions (Panel A), shows a progressive worsening of bilateral patchy infiltrates in the lower lungs with mild pleural effusion. 


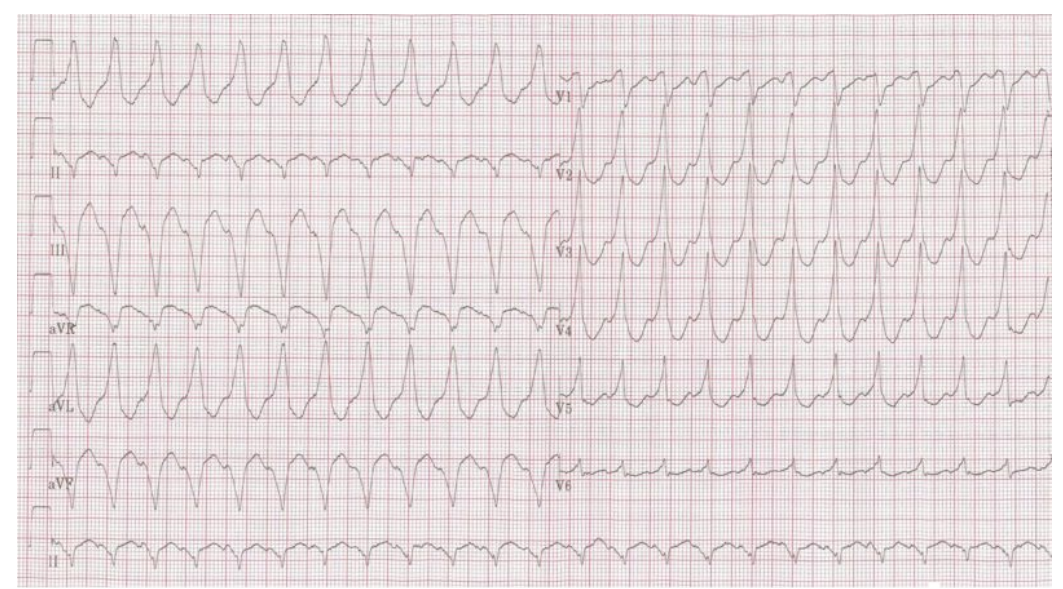

Figure 2. ECG shows sustained ventricular tachycardia.

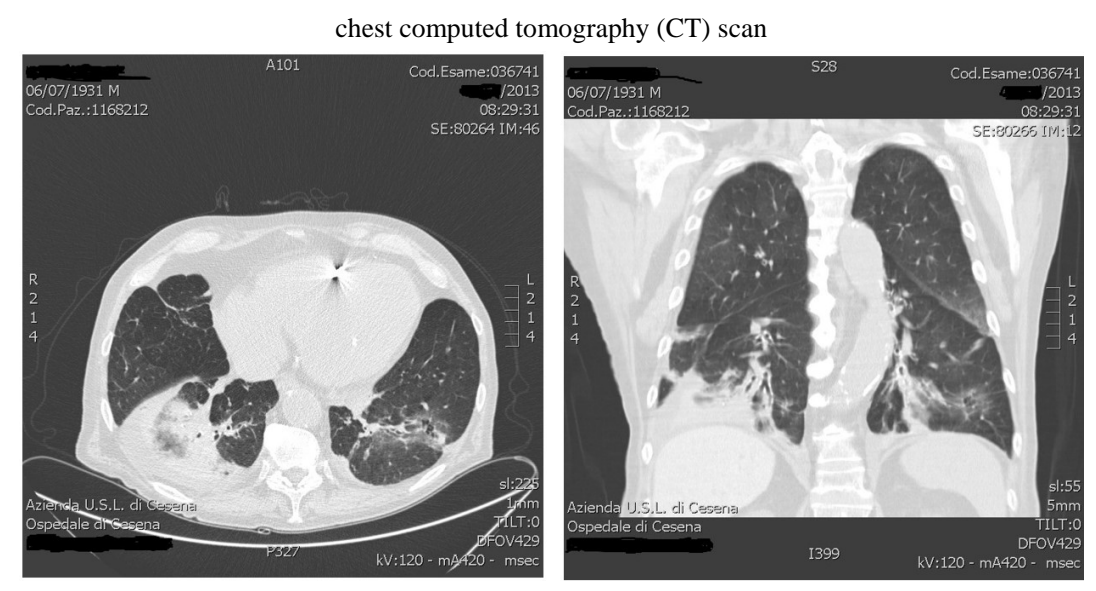

Figure 3. Chest HRCT scan shows findings similar to the chest radiograph, with bilateral areas of consolidation and ground glass opacities with a peripheral location.

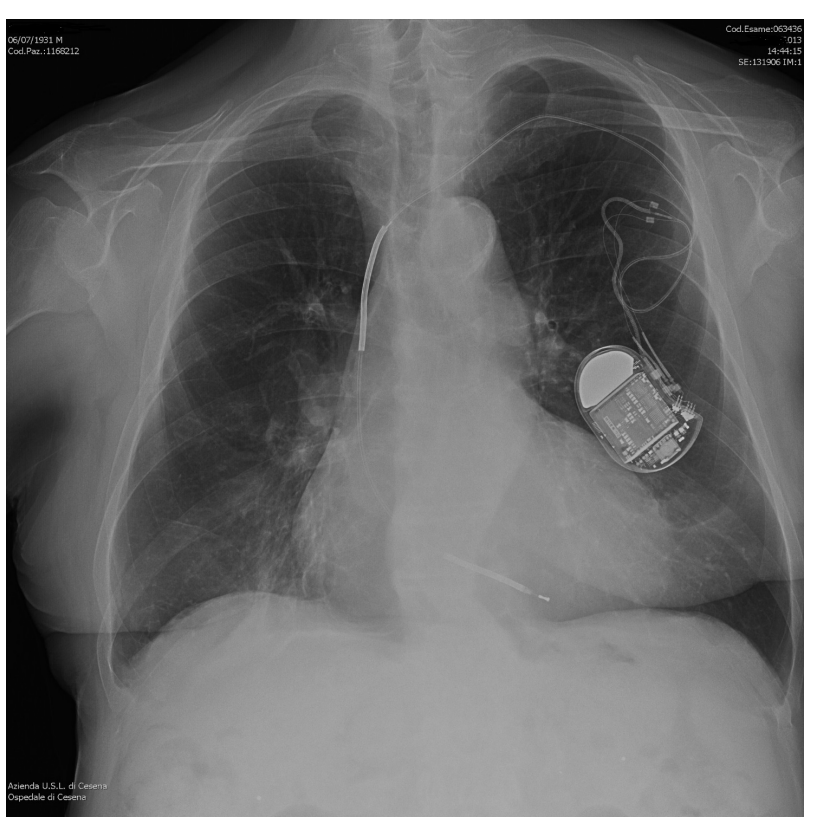

Figure 4. Chest $x$ ray shows full resolution of infiltrates with normal radiographic appearances. this in mind because medical decision depends on clinical skills rather than any definitive diagnostic tests or proven therapies. Pulmonary toxicity can be fatal. A high index of suspicion is necessary in establishing the diagnosis of amiodarone-induced BOOP, since most cases are reversible if detected early. In summary, the toxicity of amiodarone should be considered in the differential diagnosis of all patients who are being treated with this medication and presenting progressive or acute respiratory symptoms, especially those with a history of chronic lung disease, supplemental oxygen therapy and after cardiac surgery.

\section{REFERENCES}

[1] Epler, G.R., Colby, T.V., McLoud, T.C., Carrington, C.B. and Gaensler, E.A. (1985) Bronchiolitis obliterans organizing pneumonia. The New England Journal of Medicine, 312, 152-158. http://dx.doi.org/10.1056/NEJM198501173120304

[2] Epler, G.R. (2001) Bronchiolitis obliterans organizing pneumonia. Archives of Internal Medicine, 161, 158-164. 
http://dx.doi.org/10.1001/archinte.161.2.158

[3] Cordier, J.F. (1993) Cryptogenic organizing pneumonitis: bronchiolitis obliterans organizing pneumonia. Clinics in Chest Medicine, 14, 677-692.

[4] Dimopoulou, I., Marathias, K., Daganou, M., et al. (1997) Low-dose amiodarone-related complications after cardiac operations. The Journal of Thoracic and Cardiovascular Surgery, 114, 31-37.

http://dx.doi.org/10.1016/S0022-5223(97)70114-4

[5] Martin, W.J. and Rosenow, E.C. (1988) Amiodarone pulmonary toxicity: Recognition and pathogenesis. Chest, 93, 1067-1075. http://dx.doi.org/10.1378/chest.93.5.1067

[6] Costabel, U., Guzman, J. and Teschler, H. (1995) Bronchiolitis obliterans with organizing pneumonia: Outcome. Thorax, 50, S59-S64. http://dx.doi.org/10.1136/thx.50.Suppl 1.S59

[7] Murphy, J.M., Schnyder, P., Verschakelen, J., Leuenberger, P. and Flower, C.D. (1999) Linear opacities on HRCT in bronchiolitis obliterans organizing pneumonia.
European Radiology, 9, 1813-1817. http://dx.doi.org/10.1007/s003300050928

[8] Cooper Jr., J.A. (1997) Drug-induced lung disease. Advanced Internal Medicine, 42, 231-268.

[9] Nacca, N., Bhamidipati, C.M., Yuhico, L.S., Pinnamaneni, S. and Szombathy, R. (2012) Severe amiodarone induced pulmonarytoxicity. Journal of Thoracic Disease, 4, 667670.

[10] Fabiani, I., Tacconi, D., Grotti, S., et al. (2011) Amiodarone-induced pulmonary toxicity mimicking acute pulmonary edema. Journal of Cardiovascular Medicine, 12, 361-365.

http://dx.doi.org/10.2459/JCM.0b013e328334a3f4

[11] Rossi, S.E., Erasmus, J.J., McAdams, H.P., Sporn, T.A. and Goodman, P.C. (2000) Pulmonary drug toxicity: Radiologic and pathologic manifestations. RadioGraphics, 20, 1245-1259.

http://dx.doi.org/10.1148/radiographics.20.5.g00se08124 $\underline{5}$ 